\title{
Left cardiac sympathetic denervation in the Netherlands for the treatment of inherited arrhythmia syndromes
}

\author{
L. R. A. Olde Nordkamp • A. H. G. Driessen • A. Odero • \\ N. A. Blom • D. R. Koolbergen - P. J. Schwartz • \\ A. A. M. Wilde
}

Published online: 13 February 2014

(C) The Author(s) 2014. This article is published with open access at Springerlink.com

\begin{abstract}
Introduction Treating therapy-resistant patients with inherited arrhythmia syndromes can be difficult and left cardiac sympathetic denervation (LCSD) might be a viable alternative treatment option. We provide an overview of the indications and outcomes of LCSD in patients with inherited arrhythmia syndromes in the only tertiary referral centre in the Netherlands where LCSD is conducted in these patients.

Methods This was a retrospective study, including all patients with inherited arrhythmia syndromes who underwent LCSD in our institution between 2005 and 2013. LCSD involved ablation of the lower part of the left stellate ganglion and the first four thoracic ganglia.

Results Seventeen patients, 12 long-QT syndrome (LQTS) patients $(71 \%)$ and 5 catecholaminergic polymorphic ventricular tachycardia (CPVT) patients (29\%), underwent LCSD. Most patients (94\%) were referred because of therapyrefractory cardiac events. In $87 \%$ the annual cardiac event
\end{abstract}

L. R. A. Olde Nordkamp • A. A. M. Wilde $(\bowtie)$

Heart Center, Department of Cardiology, Academic Medical Centre, PO Box 22700, 1100 DE Amsterdam, the Netherlands

e-mail: a.a.wilde@amc.uva.nl

A. H. G. Driessen • D. R. Koolbergen

Heart Center, Department of Cardiothoracic Surgery, Academic

Medical Centre, Amsterdam, the Netherlands

A. Odero

Department of Lung, Blood and Heart, University of Pavia, Pavia, Italy

N. A. Blom

Department of Paediatric Cardiology, Academic Medical Centre, Amsterdam, the Netherlands

P. J. Schwartz

Center for Cardiac Arrhythmias of Genetic Origin, IRCCS Instituto

Auxologico Italiano, Milan, Italy rate decreased. However, after 2 years the probability of complete cardiac event-free survival was $59 \%$ in LQTS and $60 \%$ in CPVT patients. Two patients (12\%) had major nonreversible LCSD-related complications: one patient suffered from a Harlequin face post-procedure and one severely affected LQT8 patient died the day after LCSD due to complications secondary to an arrhythmic storm during the procedure. Conclusion LSCD for inherited arrhythmia syndromes, which is applied on a relatively small scale in the Netherlands, reduced the cardiac event rate in $87 \%$ of the high-risk patients who had therapy-refractory cardiac events, while the rate of major complications was low. Therefore, LSCD seems a viable treatment for patients with inherited arrhythmia syndromes without other options for therapy.

Keywords Left cardiac sympathetic denervation · Inherited arrhythmia syndromes $\cdot$ Ventricular arrhythmias

\author{
P. J. Schwartz \\ Department of Molecular Medicine, University of Pavia, Pavia, Italy \\ P. J. Schwartz \\ Cardiovascular Genetics Laboratory, Hatter Institute for \\ Cardiovascular Research in Africa, Department of Medicine, \\ University of Cape Town, Cape Town, South Africa \\ P. J. Schwartz \\ Department of Medicine, University of Stellenbosch, Stellenbosch, \\ South Africa \\ P. J. Schwartz \\ Department of Family and Community Medicine, College of \\ Medicine, King Saud University, Riyadh, Saudi Arabia
}

A. A. M. Wilde

Princess Al-Jawhara Albrahim Centre of Excellence in Research of Hereditary Disorders, King Abdulaziz University, Jeddah, Saudi Arabia 


\section{Introduction}

Primary inherited arrhythmia syndromes, such as long-QT syndrome (LQTS) and catecholaminergic polymorphic ventricular tachycardia (CPVT), are genetic cardiac diseases without apparent structural heart disease that may cause cardiac syncope and sudden cardiac death, mainly in young individuals. These cardiac events are generally induced by physical or emotional stress triggers [1-3]. Hence, $\beta$-blockers are considered first-line therapy in symptomatic and asymptomatic patients in both conditions $[4,5]$. In addition, an implantable cardiac defibrillator (ICD) is often used in patients who continue to have ventricular arrhythmias despite $\beta$-blocker therapy $[5,6]$. However, ICDs do not prevent ventricular arrhythmias and can even trigger catecholamine release, subsequently resulting in arrhythmic storms and even death [7]. Also the cost of frequent shocks in terms of pain and fear is substantial [8] and young patients with ICDs are more likely to experience device complications, including inappropriate shocks and lead-related complications, over many years of use [9].

In 1971, Moss and McDonald [10] described left cardiac sympathetic denervation (LCSD), which prevents norepinephrine release in the heart, therefore raising the threshold for ventricular fibrillation without reducing the heart rate or impairing myocardial contractility [11]. In the last decade LCSD has received renewed attention as a viable alternative treatment for therapy-resistant LQTS and CPVT patients. A significant protective effect of LCSD was demonstrated in both symptomatic and asymptomatic LQTS and CPVT patients [12-16]. In the Netherlands, LCSD is applied on a small scale and we hereby provide an overview of the indications and outcomes of LCSD in patients with inherited arrhythmia syndromes in the only tertiary referral centre where LCSD is conducted.

\section{Methods}

\section{Study design}

Patients who received LCSD in our hospital between 1 November 2005 and 1 February 2013 for LQTS or CPVT were included in this study. LQTS was defined according to the diagnostic criteria described by Schwartz et al. [17] The QT interval was assessed from lead II/V5 and corrected for heart rate using Bazett's formula. CPVT was diagnosed based on exerciseinduced bidirectional or polymorphic ventricular tachycardia (VT) in the absence of structural cardiac disease and a putative pathogenic mutation in a CPVT-causing gene [2].

All data available prior to and after the LCSD were retrospectively extracted from medical records. These included demographic data, clinical data (initial presentation, age at onset, family history of sudden cardiac death (SCD)
$<60$ years), genotype, ECG data, presence of ICD and medical therapy, incidence of cardiac events and indication for surgery. Cardiac events were defined as cardiac syncope, aborted cardiac arrest (ACA), appropriate ICD shocks or malignant non-sustained ventricular tachycardias (NSVT). LSCDrelated data included procedure time, length of hospital stay and postoperative complications. The surgery was considered secondary prevention if the patient had a history of cardiac events. The institutional review board of our institution waived the requirement for informed consent.

\section{Surgical procedure}

In the first group of patients $(n=6)$, the surgical procedure for LCSD started with an incision at the base of the neck (supraclavicular approach [18], performed by A.O., A.D. and D.K.), which is an extrapleural approach without opening the chest. The other patients underwent a video-assisted thoracoscopic LCSD [19], except for one 2-month-old baby who underwent denervation by thoracotomy (performed by A.D. and D.K.). In both the supraclavicular and videoassisted thoracoscopic approach, the lower part of the stellate ganglion (preferably dissection along the anatomical fusion between the upper and lower pole) was removed together with the second and third thoracic ganglia; the fourth ganglion was cauterised. Additional visible nerve structures from the sympathetic ganglia towards the heart were also cauterised. It provides adequate cardiac denervation with no or minimal Horner's syndrome, because the upper half of the stellate ganglion is preserved.

\section{Statistics}

All data were analysed with SPSS (19.0 SPSS Inc, Chicago, IL). Categorical data are displayed as percentage and compared between groups using a $\chi^{2}$ test. Normally distributed continuous data, tested for normality with the Shapiro-Wilk test, were described as mean (standard deviation [SD]). Continuous data not normally distributed were expressed as median (interquartile range [IQR]) and compared between groups using the Mann-Whitney $U$-test. Annual event rates were calculated as the number of events per year of observation. Postoperative event-free survival was described by Kaplan-Meier cumulative estimates. Confidence intervals for the median number of episodes and the median annual event rates were based on the method proposed by Bonett and Price [20]. The patient who died a few days after LCSD was removed from the denominator in the cardiac event analysis after LCSD, since follow-up was not possible. $P$ values $<0.05$ were considered statistically significant. 


\section{Results}

\section{Patient characteristics}

From November 2005 to January 2013, 17 patients (12 LQTS patients $(71 \%)$ and 5 CPVT patients $(29 \%)$ ) had a LCSD performed at our institution. Clinical characteristics of these patients are summarised in Table 1 . The mean age of the entire study population at the time of LCSD was $19 \pm 14$ years and $59 \%$ were female. All patients were on $\beta$-blocking therapy and three of the five CPVT patients had additionally taken flecainide prior to LCSD. Eight patients (47\%), of whom seven were diagnosed with LQTS, had already ICD implanted by the time of the surgery and one LQTS patient received an ICD 1 month after LCSD.

All CPVT patients had a mutation in the RyR2 gene, which were classified as putatively pathogenic in four patients. One patient (\#2 in Fig. 1) had an unclassified variant in the RyR2 gene. Of the patients with LQTS, six $(50 \%)$ had a mutation in the KCNH2 gene, and two patients (17\%) had LQTS type 1 based on a mutation in the KCNQ1 gene. One LQTS patient (\#11) was tested for all known LQTS genes, but no mutation was found.

\section{Indications}

Sixteen patients (94\%) underwent LCSD after experiencing cardiac events under adequate medical therapy (9 [53\%] VT/ VF, 5 [29 \%] syncope and 2 [12\%] malignant NSVT), while one patient $(5.9 \%)$ underwent LCSD for primary prevention because of $\beta$-blocker intolerance. Figure 1 demonstrates the event rate timing prior to LCSD.

\section{LCSD procedure}

Six (35\%) patients underwent LCSD using the supraclavicular approach and in 10 patients (58\%) LCSD was performed via a video-assisted thoracoscopic approach. One 2-month-old baby underwent LCSD via thoracotomy because a video-assisted thoracoscopic approach was not deemed feasible. The median skin-to-skin time was 46 (IQR 39-61) minutes, with a median time of 40 (IQR 34-78) minutes for the supraclavicular approach and 47 (IQR 43$67)$ minutes for the thoracoscopic approach $(p=0.33)$. The median number of hospitalisation days after the procedure was 3 (IQR 2-7) days. There was no difference in number of hospitalisation days between the two surgical approaches (supraclavicular approach 3 (IQR 3-23) days and thoracoscopic approach 2 (IQR 2-4) days; $p=0.13$ ).

Major non-reversible complications of the LCSD were reported in $2 / 17(12 \%)$ of the patients. One patient $(5.9 \%)$ suffered from a postoperative Harlequin face. One severely affected LQT8 patient (5.9\%; patient \#6 in Fig. 1) with multiple arrhythmic storms before LCSD died after the procedure, due to multi-organ failure secondary to bradycardias followed by arrhythmic storms in combination with severe electrolytic disturbances and hypoglycaemia during and after the procedure. Minor complications of the LCSD, which spontaneously resolved, were reported in four (24\%) additional patients: three of these patients (18\%), all treated using the video-assisted thoracoscopic approach, were diagnosed with a post-procedural pneumothorax and one patient $(5.9 \%)$ had a transient postoperative Horner's syndrome.

Cardiac events after LCSD

During a median follow-up of 34 (IQR 16-77) months, 7 out of $15(47 \%)$ symptomatic patients did not experience any occurrence of cardiac events after LCSD (Fig. 1). Cardiac events continued in 8/15 (53\%) symptomatic patients (Fig. 1 and Table 2). In one of these patients with recurrences, an ICD shock occurred after a decrease in $\beta$-blocker dosage and in two patients only asymptomatic NSVT persisted, while they suffered from life-threatening cardiac syncope and ICD shocks before LCSD. Additionally, one patient (\#17), who was diagnosed with LQT3 which overlapped with Brugada syndrome, had recurrences of life-threatening cardiac events after LCSD, but these events were mainly fever-related and thus probably related to Brugada syndrome. Life-threatening cardiac events persisted at a high(er) annual event rate after LCSD in only one patient. This LQT2 patient (\#12) experienced multiple appropriate ICD shocks after LCSD during the postpartum period. When analysing the video-assisted thoracic surgery footage in retrospect, the removal of the lower part of the stellate ganglion was insufficiently cranial. In general, cardiac events on adequate medical therapy recurred in 0 out of 2 LQT1 patients, three out of six LQT2 patients, two out of two LQT3 patients $(n=2 / 2)$, and two out of five of the CPVT patients.

In total, in $13 / 15$ symptomatic patients $(87 \%)$ the annual cardiac event rate decreased importantly after LCSD. The median number of cardiac events decreased from five $(95 \%$ confidence interval [CI]: $0-22)$ to 0 (95\% CI: $0-3.5)$. The median annual event rate decreased from 1.7 (95\% CI: 0-5.6) to 0 (95\% CI: 0-1.0).

Kaplan-Meier estimates of postoperative cardiac event-free survival in all patients and by disease are shown in Fig. 2. Patients with LQTS had a $71 \%$ and $59 \%$ probability of eventfree survival at 1 and 2 years, respectively, while these rates were $60 \%$ after both 1 and 2 years in CPVT patients.

\section{Discussion}

Today, LCSD is increasingly recognised as a viable treatment for therapy-resistant LQTS and CPVT patients [13-15]. Here 
Table 1 Patient characteristics

\begin{tabular}{|c|c|c|c|}
\hline & Total & LQTS & CPVT \\
\hline Total number of patients & 17 & 12 & 5 \\
\hline Female & $10(59 \%)$ & $7(58 \%)$ & $3(60 \%)$ \\
\hline $\begin{array}{l}\text { Family history of sudden cardiac } \\
\text { death }<60 \text { years }\end{array}$ & $5(29 \%)$ & $2(17 \%)$ & $3(60 \%)$ \\
\hline \multicolumn{4}{|l|}{ Genotype } \\
\hline - KCNQ1 mutation (LQT1) & $2(12 \%)$ & $2(17 \%)$ & 0 \\
\hline - KCNH2 mutation (LQT2) & $6(35 \%)$ & $6(50 \%)$ & 0 \\
\hline - SCN5A mutation (LQT3) & $2(12 \%)$ & $2(17 \%)$ & 0 \\
\hline - CACNA1C mutation (LQT8) & $1(5.9 \%)$ & $1(8.3 \%)$ & 0 \\
\hline - LQTS of unknown type & $1(5.9 \%)$ & $1(8.3 \%)$ & 0 \\
\hline - RyR2 mutation & $5(29 \%)$ & 0 & $5(100 \%)$ \\
\hline \multicolumn{4}{|l|}{ Presentation } \\
\hline - Aborted cardiac arrest / VT in history & $6(35 \%)$ & $4(33 \%)$ & $2(40 \%)$ \\
\hline - Cardiac syncope & $6(35 \%)$ & $4(33 \%)$ & $2(40 \%)$ \\
\hline - Family history & $4(24 \%)$ & $3(25 \%)$ & $1(20 \%)$ \\
\hline - Medical evaluation, other & $1(5.9 \%)$ & $1(8.3 \%)$ & 0 \\
\hline Age at first cardiac event (median) & 11 (IQR 0-18) & 12 (IQR 0-17) & 10 (IQR 2-19) \\
\hline Age at LCSD (mean) & $19 \pm 14$ & $20 \pm 16$ & $17 \pm 4$ \\
\hline \multicolumn{4}{|l|}{ Indication for LCSD } \\
\hline - Primary prevention & $1(5.9 \%)$ & $1(8.3 \%)$ & 0 \\
\hline - Secondary prevention & $16(94 \%)$ & $11(92 \%)$ & $5(100 \%)$ \\
\hline \multicolumn{4}{|l|}{ Prior therapies } \\
\hline - $\beta$-blocker & $17(100 \%)$ & $12(100 \%)$ & $5(100 \%)$ \\
\hline - Mexiletine & $2(12 \%)$ & $2(17 \%)$ & 0 \\
\hline - Flecainide & $4(24 \%)$ & $1(8.3 \%)$ & $3(60 \%)$ \\
\hline ICD & $9(53 \%)$ & $8(67 \%)$ & $1(20 \%)$ \\
\hline \multicolumn{4}{|l|}{ QTc } \\
\hline - Pre-LCSD (median) & 460 (IQR 395-496) & 477 (IQR 413-526) & 390 (IQR 381-442) \\
\hline - Post-LCSD (median) & 450 (IQR 413-504) & 459 (IQR 440-538) & 402 (IQR 348-464) \\
\hline
\end{tabular}

$C P V T$ catecholaminergic polymorphic ventricular tachycardia, ICD implantable cardiac defibrillator, IQR interquartile range, $L C S D$ left cardiac sympathetic denervation, LQTS long-QT syndrome

Fig. 1 Comparison of cardiac events before and after LSCD

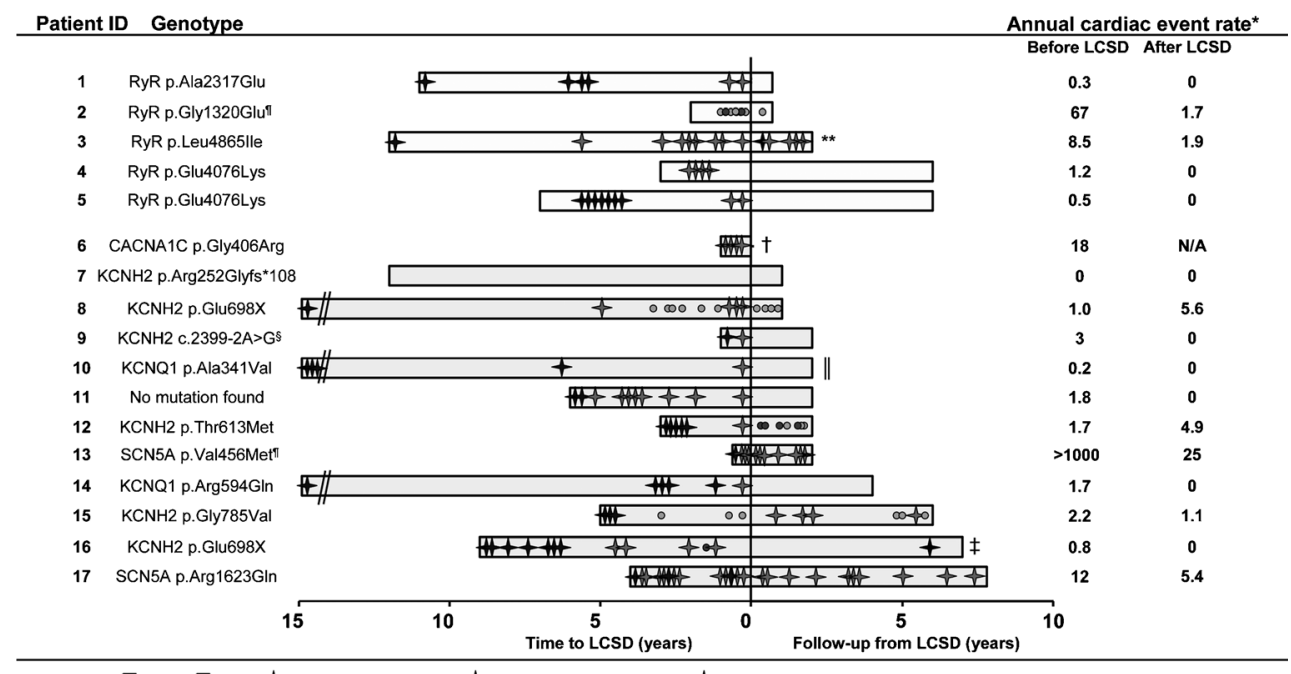

$\square$ CPVT $\square$ LQTS + Syncope/ACA on therapy + Syncope/ACA not on therapy + Syncope/ACA with remark $\bullet$ Symptomatic NSVT ${ }^{\circ}$ Asymptomatic NSVT *on adequate therapy: * * $1 \times$ shock on SVT causing VT, next shock sinus rnythm; † patient deceased after LCSD; $\pi$ VUS2;
$\S$ splice site mutation; $\|$ multiple syncope episodes during basketball at age 13-24 years; $\neq 1 \times$ shock on VT after lowering the $\beta$-blocker dosage 
Table 2 Patient characteristics of patients with cardiac events after LCSD while on adequate medical therapy

\begin{tabular}{lllllllll}
\hline $\begin{array}{l}\text { Patient } \\
\text { ID }\end{array}$ & Sex & Disease & $\begin{array}{l}\text { Age of } \\
\text { onset }\end{array}$ & $\begin{array}{l}\text { Age } \\
\text { LCSD }\end{array}$ & $\begin{array}{l}\text { Number of CE } \\
\text { before LCSD }\end{array}$ & $\begin{array}{l}\text { Number of CE } \\
\text { after LCSD }\end{array}$ & $\begin{array}{l}\text { Event } \\
\text { circumstance }\end{array}$ & $\begin{array}{l}\text { QTc before/ } \\
\text { after LCSD }\end{array}$ \\
\hline 2 & Female & CPVT & 21 & 21 & $>100^{\mathrm{b}}$ & $1^{\mathrm{b}}$ & Exercise & $383 / 423 \mathrm{~ms}$ \\
3 & Male & CPVT & 3 & 15 & $>100$ & 4 & Exercise & During rest, waking up or in the evening \\
8 & Female & LQT2 & 16 & 35 & $>10$ & $7^{\mathrm{b}}$ & 460/441 ms \\
12 & Female & LQT2 & 16 & 19 & 3 & $>10^{\mathrm{b}}$ & Sudden emotion/noise & N33/439 ms \\
13 & Male & LQT3 & Birth & 0 & $>100$ & $>50$ & Neonatal, during rest or suddenly \\
15 & Female & LQT2 & 20 & 25 & 9 & 7 & Sudden emotion/noise and postpartum period & $506 / 503 \mathrm{~ms}$ \\
16 & Female & LQT2 & 12 & 21 & 5 & $1^{\mathrm{c}}$ & During rest, waking up or suddenly & $485 / 505 \mathrm{~ms}$ \\
17 & Male & LQT3 and BrS & Birth & 4 & $>50$ & $>10$ & Neonatal, playing and during fever & $535 / 352 \mathrm{~ms}$ \\
\hline
\end{tabular}

$B r S$ Brugada syndrome, CPVT catecholaminergic polymorphic ventricular tachycardia, LCSD left cardiac sympathetic denervation, $L Q T S$ long-QT syndrome

${ }^{a}$ Under adequate medication

${ }^{\mathrm{b}}$ Only short non-sustained ventricular tachycardia $(<10$ complexes)

${ }^{\mathrm{c}}$ Cardiac event (CE) after LCSD was after decrease in $\beta$-blocker dosage

we report the experience in the only tertiary referral centre in the Netherlands for this treatment in 17 patients with inherited arrhythmia syndromes. Most patients (94\%) were referred because of therapy-refractory cardiac events. In $87 \%$ of the symptomatic patients, the annual cardiac event rate decreased. However, after 2 years the probability of cardiac event-free survival was $59 \%$ in LQTS and $60 \%$ in CPVT patients. There
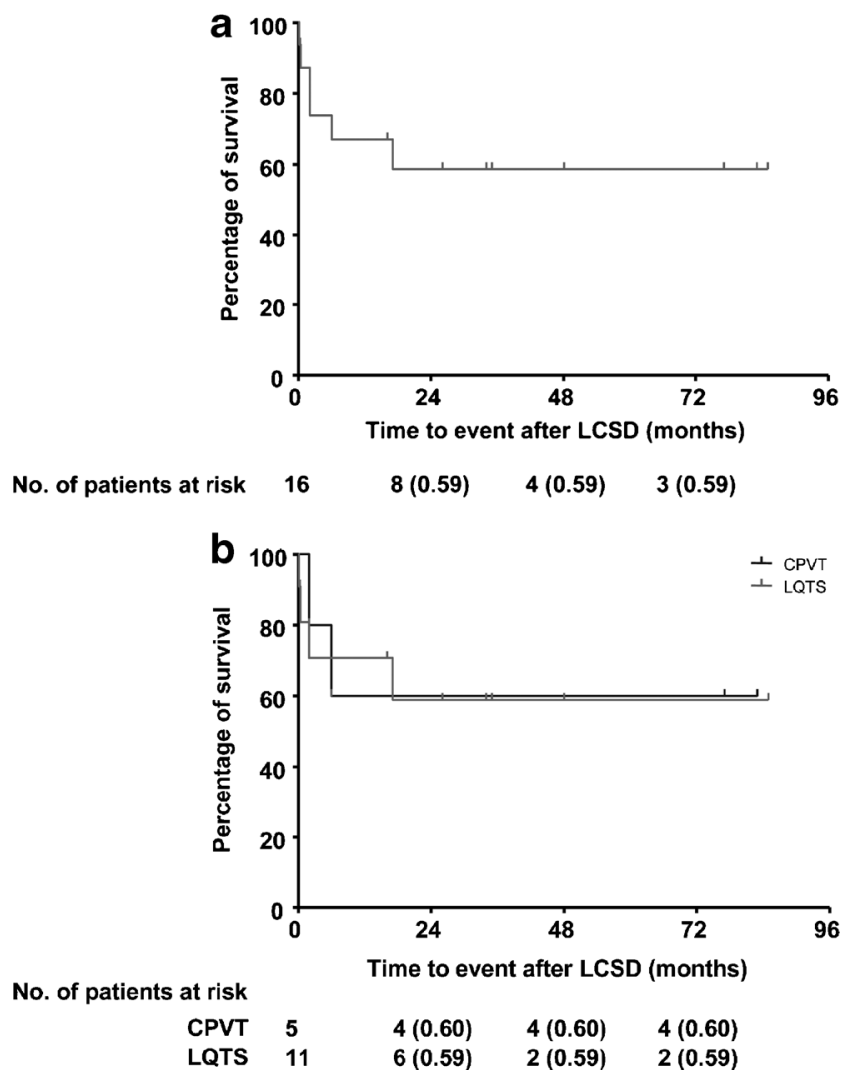

Fig. 2 a Kaplan-Meier curves of event-free survival after LCSD: a all patients and $\mathbf{b}$ by disease were four patients (24\%) with minor reversible complications who did not require any intervention, which is a similar number compared with other centres [14, 15, 21], and one patient $(5.9 \%)$ with a non-reversible post-procedural Harlequin face. Also, one LQT8 patient (5.9 \%) died after the LCSD procedure due to complications secondary to bradycardias followed by an arrhythmic storm in combination with electrolytic disturbances and hypoglycaemia during the procedure. An identical case was reported by Schwartz et al. [12] in 1991, where a 3-yearold female patient died early postoperatively with suspected post-anaesthesia hypoxic distress (hypoglycaemia, respiratory failure, complete heart block and asystole). This patient was later identified as a LQT8 patient, and extreme caution should be taken in this type of patients.

\section{Success of LCSD}

Our results are in agreement with previously published experience. Indeed, multiple studies have reported a significant decrease of cardiac events in $90 \%$ of the symptomatic patients with inherited arrhythmia syndromes after LCSD, although approximately $40 \%$ remained symptomatic [13-15, 19, 21-23]. The antiarrhythmic and antifibrillatory effects of LCSD are attributed to the reduced release of norepinephrine at the level of the ventricles [11]. This sympathetic blockade prolongs ventricular refractoriness and increases the ventricular fibrillation threshold, resulting in the prevention or suppression of triggered activity [11]. The denervation has a highly specific antiarrhythmic effect, without reducing the heart rate (which might be especially important for LQT3 patients) and without impairing myocardial contractility [11]. Additionally, because the denervation does not completely eliminate catecholamine input to the heart, surgery does not lead to hypersensitivity [11]. And lastly, re-innervation is not 
expected because the denervation is pre-ganglionic, which makes it likely that the effects are durable [18]. However, especially in highly symptomatic patients with inherited arrhythmia syndromes, LCSD has so far been advocated merely as an adjuvant therapy besides $\beta$-blockers (in LQTS and CPVT) or flecainide (in CPVT), as also demonstrated by the breakthrough cardiac event of patient \#16 after lowering the $\beta$-blocker dosage. Indeed, ICDs should still be considered in patients at high risk for SCD.

Recently, percutaneous renal sympathetic denervation has emerged as a therapeutic option for patients with hyperactivity of the sympathetic system such as therapy-resistant hypertension [24]. Additionally, in animal models renal denervation suppressed ventricular arrhythmias [25]. These findings support the hypothesis that renal denervation might also be useful in reducing sympathetic activity in highly symptomatic patients with inherited arrhythmia syndromes and thereby reducing cardiac event rate, although this needs to be studied in the future.

Response to LCSD among the different genotypes

Although there was a significant decrease in cardiac events in most patients, half of the patients remained symptomatic. Post-LCSD cardiac events recurred in $3 / 6$ of the LQT2 patients, $2 / 2$ of the LQT3 patients, and 2/5 of the CPVT patients, while none of the LQT1 patients had events. The number of patients is too small to draw any meaningful conclusions, but the various results among the different genotypes are in agreement with the underlying pathophysiological mechanism. During gradual progressive sympathetic activation (such as exercise), the reduced, catecholamine-sensitive, slow delayed rectifier potassium current $\left(\mathrm{I}_{\mathrm{Ks}}\right)$ in LQT1 patients prevents the necessary QT adaptation, causing a risk for triggered activity and ventricular arrhythmias [26, 27]. Therefore, removing the sympathetic activation of the heart with LCSD appeared to be very successful in LQT1 patients [15].

On the other hand, cardiac events in LQT2 patients are often triggered by a sudden heart rate acceleration, such as during a loud noise or surprising emotion [28]. In LQT2 patients with a reduced rapid delayed rectifier potassium current $\left(\mathrm{I}_{\mathrm{Kr}}\right)$, possible extrasystoles triggered by these triggers result in a markedly pause-dependent prolongation of the action potential duration of the following heart beat and attendant risk of early afterdepolarisations [27, 29]. Sudden heart rate increment is caused by vagal withdrawal rather than sympathetic activation [30] and this might explain that removal of the sympathetic activation by LCSD is not so successful in all LQT2 patients. Also the occurrence of extrasystoles might not be prevented.

In both LQT3 patients, the number of cardiac events decreased, but the effect of LCSD was not complete. Also, failure of complete LCSD success in CPVT may be attributed to the fact that only the local, cardiac, release of catecholamines is blocked. Systemic release of catecholamines is still pertinent and may be sufficient to elicit triggered arrhythmias during physical exercise, despite $\beta$-blockade and flecainide therapy. Furthermore, one CPVT patient (\#2) with recurrent events carries an unclassified variant in the RyR2 gene, which might indicate that the diagnosis CPVT might not be correct.

Although the success of LCSD was not complete among the different genotypes, the number of events decreased in almost all patients (87\%), thus significantly improving the quality of life of the patients and their families. Therefore, LCSD continues to be worthwhile in highly symptomatic patients with primary arrhythmias.

\section{Limitations}

This is retrospective study with a small number of patients, secondary to the rare prevalence of therapy-resistant LQTS and CPVT patients. Due to the retrospective nature, a quantitative marker of arrhythmia burden has been difficult to express in statistically comparable data due to the large variability in presenting symptoms, resulting in a wide definition of cardiac events. Also, in patients without an ICD, the lack of continuous heart rate monitoring could have resulted in an underestimation of cardiac events, especially NSVT. However, cardiac event monitoring before and after LCSD was identical in all patients except for one LQT patient who received an ICD 1 month after LCSD.

\section{Conclusion}

LSCD for inherited arrhythmia syndromes, although applied on a small scale in the Netherlands, reduced the cardiac event rate in $87 \%$ of the high-risk patients who had therapy refractory cardiac events. Although one severely affected LQT8 patient died secondary to surgery-related issues, LSCD seems a viable treatment for patients with inherited arrhythmia syndromes without other options for therapy.

Funding None.

Conflict of interest None declared.

Open Access This article is distributed under the terms of the Creative Commons Attribution License which permits any use, distribution, and reproduction in any medium, provided the original author(s) and the source are credited.

\section{References}

1. Schwartz PJ, Priori SG, Spazzolini C, et al. Genotype-phenotype correlation in the long-QT syndrome: gene-specific triggers for lifethreatening arrhythmias. Circulation. 2001;103:89-95. 
2. Leenhardt A, Denjoy I, Guicheney P. Catecholaminergic polymorphic ventricular tachycardia. Circ Arrhythm Electrophysiol. 2012;5: 1044-52.

3. Amin AS, Klemens CA, Verkerk AO, et al. Fever-triggered ventricular arrhythmias in Brugada syndrome and type two long-QT syndrome. Neth Heart J. 2010;18:165-9.

4. Moss AJ, Zareba W, Hall WJ, et al. Effectiveness and limitations of beta-blocker therapy in congenital long-QT syndrome. Circulation. 2000;101:616-23.

5. van der Werf C, Zwinderman AH, Wilde AA. Therapeutic approach for patients with catecholaminergic polymorphic ventricular tachycardia: state of the art and future developments. Europace. 2012;14: 175-83.

6. Schwartz PJ, Spazzolini C, Priori SG, et al. Who are the long-QT syndrome patients who receive an implantable cardioverterdefibrillator and what happens to them? Data from the European Long-QT Syndrome Implantable Cardioverter-Defibrillator (LQTS ICD) registry. Circulation. 2010;122:1272-82.

7. Mohamed U, Gollob MH, Gow RM, et al. Sudden cardiac death despite an implantable cardioverter-defibrillator in a young female with catecholaminergic ventricular tachycardia. Heart Rhythm. 2006;3:1486-9.

8. Sears SF, St Amant JB, Zeigler V. Psychosocial considerations for children and young adolescents with implantable cardioverter defibrillators: an update. Pacing Clin Electrophysiol. 2009;32 Suppl 2: S $80-2$

9. Olde Nordkamp LR, Wilde AA, Tijssen JG, et al. The ICD for primary prevention in patients with inherited cardiac diseases: indications, use, and outcome: a comparison with secondary prevention. Circ Arrhythm Electrophysiol. 2013;6:91-100.

10. Moss AJ, McDonald J. Unilateral cervicothoracic sympathetic ganglionectomy for the treatment of long QT interval syndrome. N Engl J Med. 1971;285:903-4.

11. Schwartz PJ. The rationale and the role of left stellectomy for the prevention of malignant arrhythmias. Ann N Y Acad Sci. 1984;427: 199-221.

12. Schwartz PJ, Locati EH, Moss AJ, et al. Left cardiac sympathetic denervation in the therapy of congenital long QT syndrome. A worldwide report. Circulation. 1991;84:503-11.

13. Schwartz PJ, Priori SG, Cerrone M, et al. Left cardiac sympathetic denervation in the management of high-risk patients affected by the long-QT syndrome. Circulation. 2004;109: 1826-33.

14. Collura CA, Johnson JN, Moir C, et al. Left cardiac sympathetic denervation for the treatment of long QT syndrome and catecholaminergic polymorphic ventricular tachycardia using video-assisted thoracic surgery. Heart Rhythm. 2009;6:752-9.

15. Bos JM, Bos KM, Johnson JN, Moir C, Ackerman MJ. Left cardiac sympathetic denervation in long QT syndrome: analysis of therapeutic non-responders. Circ Arrhythm Electrophysiol. 2013;6(4):70511 .
16. Wilde AA, Bhuiyan ZA, Crotti L, et al. Left cardiac sympathetic denervation for catecholaminergic polymorphic ventricular tachycardia. N Engl J Med. 2008;358:2024-9.

17. Schwartz PJ, Crotti L. QTc behavior during exercise and genetic testing for the long-QT syndrome. Circulation. 2011;124:2181-4.

18. Odero A, Bozzani A, De Ferrari GM, et al. Left cardiac sympathetic denervation for the prevention of life-threatening arrhythmias: the surgical supraclavicular approach to cervicothoracic sympathectomy. Heart Rhythm. 2010;7:1161-5.

19. Atallah J, Fynn-Thompson F, Cecchin F, et al. Video-assisted thoracoscopic cardiac denervation: a potential novel therapeutic option for children with intractable ventricular arrhythmias. Ann Thorac Surg. 2008;86:1620-5.

20. Bonett DG, Price RM. Statistical inference for a linear function of medians: confidence intervals, hypothesis testing, and sample size requirements. Psychol Methods. 2002;7:370-83.

21. Coleman MA, Bos JM, Johnson JN, et al. Videoscopic left cardiac sympathetic denervation for patients with recurrent ventricular fibrillation/malignant ventricular arrhythmia syndromes besides congenital long-QT syndrome. Circ Arrhythm Electrophysiol. 2012;5: $782-8$.

22. Li J, Liu Y, Yang F, et al. Video-assisted thoracoscopic left cardiac sympathetic denervation: a reliable minimally invasive approach for congenital long-QT syndrome. Ann Thorac Surg. 2008;86:1955-8.

23. Schneider HE, Steinmetz M, Krause U, et al. Left cardiac sympathetic denervation for the management of life-threatening ventricular tachyarrhythmias in young patients with catecholaminergic polymorphic ventricular tachycardia and long QT syndrome. Clin Res Cardiol. 2013;102:33-42.

24. Esler MD, Krum H, Sobotka PA, et al. Renal sympathetic denervation in patients with treatment-resistant hypertension (The Symplicity HTN-2 Trial): a randomised controlled trial. Lancet. 2010;376:19039.

25. Linz D, Wirth $\mathrm{K}$, Ukena $\mathrm{C}$, et al. Renal denervation suppresses ventricular arrhythmias during acute ventricular ischemia in pigs. Heart Rhythm. 2013;10:1525-30.

26. Schwartz PJ, Vanoli E, Crotti L, et al. Neural control of heart rate is an arrhythmia risk modifier in long QT syndrome. J Am Coll Cardiol. 2008;51:920-9.

27. Tan HL, Bardai A, Shimizu W, et al. Genotype-specific onset of arrhythmias in congenital long-QT syndrome: possible therapy implications. Circulation. 2006;114:2096-103.

28. Wilde AA, Jongbloed RJ, Doevendans PA, et al. Auditory stimuli as a trigger for arrhythmic events differentiate HERG-related (LQTS2) patients from KVLQT1-related patients (LQTS1). J Am Coll Cardiol. 1999;33:327-32.

29. Tamargo J, Caballero R, Gomez R, et al. Pharmacology of cardiac potassium channels. Cardiovasc Res. 2004;62:9-33.

30. Martin CE, Shaver JA, Leon DF, et al. Autonomic mechanisms in hemodynamic responses to isometric exercise. J Clin Invest. 1974;54:104-15. 